\title{
Correction to: Faithful qubit transmission in a quantum communication network with heterogeneous channels
}

\author{
$\mathrm{Na}$ Chen ${ }^{1}$ (D) Lin Xi Zhang ${ }^{2} \cdot$ Chang Xing $\mathrm{Pei}^{2}$ \\ Published online: 1 February 2020 \\ ๑) Springer Science+Business Media, LLC, part of Springer Nature 2020
}

\section{Correction to: Quantum Inf Process (2018) 17: 79 https://doi.org/10.1007/s11128-018-1843-8}

The original version of this article unfortunately contained a spelling mistake in the first author's affiliation. The university name "Shangdong University of Science and Technology" should be amended to "Shandong University of Science and Technology". The correct affiliations are given below.

Publisher's Note Springer Nature remains neutral with regard to jurisdictional claims in published maps and institutional affiliations.

The original article can be found online at https://doi.org/10.1007/s11128-018-1843-8.

$\mathrm{Na}$ Chen

na_chen_xd@126.com

1 College of Electronics, Communications and Physics, Shandong University of Science and Technology, Qingdao 266590, China

2 The State Key Laboratory of Integrated Services Networks, Xidian University, Xi' an 710071, China 